\title{
Presentation of integrals in introductory physics textbooks
}

\author{
Anthony Pina, Michael Loverude \\ Physics, California State University Fullerton, 800 N State College Blvd., Fullerton, CA, 92831
}

Setting up an integral based on a given physical system can be a significant barrier to success for students (Cui et. al. 2006). Prior research has shown that the way students think about integration (e.g. area under the curve, the antiderivative, or adding up pieces) before they start a problem has an effect on their success when they're constructing the necessary integrals (Jones 2015a). We examined five introductory physics textbooks, documented each example of an integral, and coded them into four categories. Area under the curve is the understanding fostered by calculus courses, but it is the least productive and one of the least utilized by physics textbooks. 


\section{BACKGROUND AND MOTIVATION}

This work is one of several studies being done at California State University Fullerton as part of an investigation of student use of math in physics courses. The findings reported in this paper come from one part of a single study broadly examining the role of integration in introductory physics courses.

\section{A. Prior research}

Many scholars in the Physics Education Research (PER) community have examined student use of mathematics in physics, focusing on mathematical reasoning and quantitative skills. Work has focused both on specific mathematical ideas in the context of physics tasks and on general models for student use of mathematics in physics. Several studies have explored student thinking on integration [1-5]. In many cases, this work complements Research in Undergraduate Math Education (RUME) findings [6-10] using applied math contexts, but only a subset of these studies acknowledge or use the RUME findings.

Meredith and Marrongelle demonstrated that students used Sherin's symbolic forms (a way of thinking about physics that associates a conceptual schema with a pattern of symbols in an equation [11]) recall, dependence, and partsof-a-whole to cue the need for integration while solving physics problems [12]. They claim that the transfer of skills from math to physics is difficult for students because it requires the reinterpretation of the math in various physical contexts. They also claim that in calculus courses integration is most frequently used to find area under the curve or the total amount of something. Student responses indicated that the parts- of- a- whole symbolic cue led to more productive work by students than the area under the curve cue. The data also showed that the parts-of-a-whole symbolic form does not cue integration for most students.

It is well established that students struggle with integration in physics problems and the cue for integration is only one of several barriers students face. Cui et al. found that students could transfer their learning from mathematics courses and apply it to physics problems, but had trouble identifying the appropriate variables and limits of integration. The same students also had had trouble identifying the circumstances that required the use of calculus. They tended to rely on simple algebraic equations instead of invoking calculus without having a complete understanding of the assumptions that yield the simple equations [13].

Students leave their calculus courses with a correct but incomplete understanding of the meaning of a definite integral. Sealy found support for the claim that understanding a definite integral as area under the curve is not sufficient on its own for students to understand a definite integral [6]. She does not claim that this understanding has a negative impact on students, only that other understandings are necessary.

There are several ways a student can think about an integral. Jones found three emergent student conceptualizations of both pure math and contextualized definite integrals [7]. The classifications identified by Jones were perimeter and area (PA), the antiderivative (AD), and adding up pieces (AUP). He claims that some student difficulties are the result of the activation of a less productive resource.

Jones further examined the extent to which the different conceptualizations of a definite integral allow students to be productive when solving physics problems [8]. Students were interviewed in pairs and asked to work through conceptual questions from both mathematics and physics contexts. The productiveness of the different conceptualizations for each episode was then determined based on whether or not it was useful, helpful, or beneficial to the task of making sense of the integrals. Students in the study who demonstrated an adding up pieces conceptualization of the contextualized integrals were more productive while working on the engineering/physics problems than those with the other conceptualizations. The least productive conceptualizations were $\mathrm{PA}$ and $\mathrm{AD}$ [8].

Jones reported a strong preference for the area and antiderivative conceptions. Students use the AUP conceptualization more frequently in physics than in mathematics, but the area and antiderivative conceptualizations are still very prevalent [9].

\section{B. Motivation for the study}

The previous work in RUME and PER makes it clear that integration in physics is a challenge for students and that the way they think about those integrals before beginning a problem affects their performance. However, it is not clear how students encounter integrals in physics instruction, and whether that instruction supports what is known of student thinking. These constraints led to our research question: how do introductory physics textbooks frame integration? Our hope was that analyzing these texts would shed some light on sources of student difficulties. We acknowledge that students do not generally read these texts cover to cover, nor is the textbook the sole source of material that is taught in physics courses. However, the textbook generally guides the instructor and the way material is framed in a textbook likely has some effect on the way material is presented in class. Homework is often drawn from the textbook and although not all students read the textbook, many consult examples for the homework [14]. The extent to which the textbook informs a class may vary, but we do believe this framing is nonetheless an important factor in the way that students learn physics, in particular for students who rely heavily on the text as a primary resource in their learning. 
TABLE I. Coding scheme: The categorizations are the fields into which integrals were coded. With them are the criteria that we used to determine a code for each integral. There was more nuance in texts, but these are the general themes and ideas that were present. The third column has specific examples of how the different codes were presented in textbooks.

\begin{tabular}{|c|c|c|}
\hline Categorization & Keywords and Ideas & Example \\
\hline Perimeter and area & $\begin{array}{l}\text { - Area referenced in the text } \\
\text { - Shaded region on graph }\end{array}$ & \\
\hline Antiderivative & $\begin{array}{l}\text { - Indefinite Integral } \\
\text { - Start and end with a function } \\
\text { - Use of constant of integration }\end{array}$ & $\begin{aligned} U & =\int \mu B \sin \theta d \theta \\
& =-\mu B \cos \theta+C\end{aligned}$ \\
\hline Adding up Pieces & $\begin{array}{l}\text { - Summation Notation } \\
\text { - Infinitesimal divisions } \\
\text { - Summation referenced in text }\end{array}$ & $\begin{aligned} \lim _{N \rightarrow \infty} \sum_{i=1}^{N} \frac{Q}{4 \pi \varepsilon_{0} L} \frac{r \Delta y}{\left(y_{i}^{2}+r^{2}\right)^{1 / 2}} \\
\frac{Q}{4 \pi \varepsilon_{0} L} \int_{-L / 2}^{L / 2} \frac{r d y}{\left(y_{i}^{2}+r^{2}\right)^{1 / 2}}\end{aligned}$ \\
\hline Procedural & $\begin{array}{l}\text { - Integration used as a tool } \\
\text { - Deriving an algebraic expression } \\
\text { - Solving differential equation }\end{array}$ & $\begin{array}{c}\int_{Q_{0}}^{Q} \frac{d Q}{Q}=-\frac{1}{R C} d t \\
Q=Q_{0} e^{-t / R C}\end{array}$ \\
\hline
\end{tabular}

\section{METHODS}

Five commonly-used introductory physics textbooks were selected for analysis: Halliday, Resnick, and Walker (HRW), Giancoli, Young and Freedman (Y\&F), Knight, and Serway and Jewett (S\&J) [15-19].

We reviewed the sections of the textbook corresponding to the material taught in our introductory mechanics and electricity and magnetism courses. In part that was due to potential differences in course coverage. This institution's third semester introductory physics course covers wave mechanics, optics, and modern physics but others may differ. We felt the topics in introductory mechanics and electricity and magnetism courses tend to be more universal. We identified all integrals presented in the relevant portions of the text (Chapters 2-11, 13, 15, 21-32 in the case of HRW).

In addition to the symbolic form of the integrals themselves, we read the corresponding introductory text to understand the context in which the integral was being presented. These integrals were then tentatively classified into the three Jones conceptualizations based on how they were presented to students ${ }_{2}$ and this classification was used as the basis of a coding scheme.

\section{A. Categorizations}

The original coding scheme was based directly on the conceptualizations detailed by Jones. To establish inter-rater reliability, the scheme and texts were given to other undergraduate research students for coding. While the different coders agreed on approximately $90 \%$ of the codes assigned, this task revealed some limitations of the original scheme. The Jones conceptualizations are meant to describe student thinking on integration, and do so well, but were not a perfect match for all of the integrals presented in textbooks. Several of the integrals did not fit any of the schemes but did appear to have elements in common; namely the integrals were definite but did not meet the criterion to be categorized as either perimeter and area or adding-up-pieces. The recent work of Bajracharya and Thompson that reported an epistemic game called analytical derivation seemed to provide a new framing for these integrals. In this game students would manipulate mathematical expressions in order to achieve some desired result, often without a strong conceptual understanding of what their work meant [20]. As a result, we added a fourth category to the coding scheme, which we dubbed procedural integrals, and recoded the texts.

In the sections below, we describe the four categories in the coding scheme with criteria and examples. A summary of the four categories with key words and ideas is in Table I.

\section{Adding up Pieces}

In Jones' work this conceptualization is used when students express or describe an integral as some sort of sum. This is a common practice in physics and engineering problems, e.g., adding small amounts of work done along a path or adding small contributions to an electric field from a larger distribution. This conceptualization is inherently connected to the underlying concept of a Reimann sum but 
TABLE II. The texts and the number of times each representation was used.

\begin{tabular}{|c|c|c|c|c|c|c|}
\hline $\begin{array}{c}\text { Textbook/ } \\
\text { Categorization }\end{array}$ & Perimeter \& Area & Antiderivative & Adding up Pieces & Procedural & Uncoded & Total \\
\hline Halliday & 4 & 3 & 27 & 36 & 9 & 79 \\
\hline Giancoli & 5 & 4 & 50 & 11 & 0 & 70 \\
\hline Young & 4 & 1 & 38 & 12 & 3 & 58 \\
\hline Knight & 6 & 0 & 29 & 11 & 5 & 51 \\
\hline Serway Jewett & 2 & 1 & 43 & 23 & 4 & 73 \\
\hline Totals & $21(6.34 \%)$ & $9(2.72 \%)$ & $187(56.50 \%)$ & $93(28.10 \%)$ & $21(6.34 \%)$ & 331 \\
\hline
\end{tabular}

has additional layers that a student must be aware of [6]. The addition of context to an integration problem makes the Reimann view insufficient to have a full understanding of the solution to the problem [6]. Integrals were coded into this category when the text transitioned to an integral by taking a limit of a sum or when summations were referenced in the accompanying text. This category also included integrals that either the text or a diagram explained by dividing an object into infinitesimally small pieces and summing all of them with the integral.

\section{Perimeter and Area}

This is the conceptualization most likely to be demonstrated by students after having completed their introductory calculus classes [6,9]. When integration is first introduced in calculus textbooks it is common to motivate its use by asking students how they would find the area under some arbitrary curve [21]. The desired result of most problems students are given when learning integration is for them to utilize it to find the area under a curve.

Integrals presented with an explicit reference to an area or shown with a graph on which there is a single shaded region were coded with the area conceptualization. Integrals that emerged from taking the limit of a sum, referenced a Reimann sum, or referenced an infinitesimal quantity were coded as adding up pieces.

\section{Antiderivative}

In the context of this paper the antiderivative category is one in which the integral is seen as an operator which takes a function as its input and whose output is another function. Necessarily this can only be applied to an indefinite integral as the output of a definite integral would not yield a function. When the text explicitly referenced an anti-derivative, depicted an indefinite integral, used generic functions in an integral, or included a constant of integration in the solution to the integral they were coded under the anti-derivative conceptualization.

\section{Procedural}

This category is adapted from the analytical derivation epistemic game described by Bajracharya and Thompson. While analytical derivation was intended to describe a strategy students use while working through math based physics problems, this was a use of integration we saw presented in physics textbooks. In their work, integration was a tool being used by students to achieve some end result, usually an algebraic relationship. The textbooks do this as well, depicting an integral as a necessary step in the derivation of an equation. We also included integrals used to solve simple differential equations in this category. This is distinct from the antiderivative category as it does not include indefinite integrals. Treating the integral as something procedural focuses less on a functional input to an integral and more on the integral acting as an operator to further a derivation.

\section{DISCUSSION}

It is important to note that while prior research in both the mathematics and physics education communities has focused primarily on definite integrals, we were coding all instances of integration in the text. Texts would often introduce an indefinite integral and later add bounds. This is distinct from the treatment of integrals in mathematics where one does not typically mix the two types of integrals in a single problem.

As shown in Table II, there were between 50 and 80 integrals in the appropriate sections of all of the texts, with 331 coded integrals all told. This number corresponds to approximately 3 or 4 integrals per chapter though these integrals are not evenly distributed across the text. Integration is a strong focus in all of the texts. Of the 331 integrals, 93\% were coded as one of our four categories.

By a significant margin the most common presentation of an integral was framed as some sort of summation and categorized as adding up pieces; 187/331 codes assigned were in this category. The second most common presentation 
was purely procedural integration with 93/331 codes being assigned to this category. These two categories account for $85 \%$ of the total codes assigned. This is a sharp contrast to the perimeter and area and antiderivative categories which together only account for $9 \%$ of the total codes assigned, in spite of this being the understanding of an integral fostered by calculus courses. There were as few as two integrals in a single textbook framed in the context of perimeter and area.

There was little variation among the different texts as far as the contexts in which integration was utilized, however, some textbooks had more examples for a single type of integral than others which led to different totals for each text. In order to be sure that our results were representative, we performed a variation of the analysis. Each topic was considered a single data point, regardless of how many examples of integration there might be in that topic. For example, a section on electrical potential with six integrals, five of which were AUP and one which was procedural; this would be codes as AUP. The results of these analyses are summarized in Table III.

TABLE III. Data coded by topic.

\begin{tabular}{ccccc}
\hline \hline $\begin{array}{c}\text { Perimeter and } \\
\text { Area }\end{array}$ & $\begin{array}{c}\text { Antide- } \\
\text { rivative }\end{array}$ & $\begin{array}{c}\text { Adding } \\
\text { up Pieces }\end{array}$ & $\begin{array}{c}\text { Proce- } \\
\text { dural }\end{array}$ & Uncoded \\
\hline 7 & 8 & 58 & 29 & 2 \\
\hline \hline
\end{tabular}

While this analysis does significantly decrease the number of integrals in total, we see that some important trends continue. The perimeter and area and antiderivative categorizations are still the least common presentations of integrals in the textbooks while adding up pieces and procedural integrals still make up more than $82 \%$ of those presented. We note again that this in stark contrast to the results of instruction in calculus classes, in which students emerge with a strong association between integration and area and without a strong connection to adding up pieces.

While quantitatively there is not much difference between the different texts, we observed some qualitative features in Knight that make it distinct from the other texts analyzed. There are some instances when the text in Knight presents a concept as a sum where others would have transitioned to an integral expression. Knight also takes the time to explain/teach some calculus concepts before they are utilized in physics problems. For example, it has a section dedicated to the notion of a line integral leading up to the introduction of Ampere's law. There are also times when
Knight notes explicitly in the text that calculus is new to students in the course and takes the time to go through steps other textbooks are likely to gloss over in solving an integral. Knight also tends to focus on the physics the math is explaining more than the math leading to more integrals that were difficult to categorize. Area appears more in Knight than in any of the other texts.

Knight is also the most explicit about its use of adding up pieces as a way to think about an integral. When presenting integrals to students in this fashion, Knight begins by presenting the students a discretized version of a problem and taking the limit of a sum to yield the integral expression as an interpretation of the physical system.

\section{IMPLICATIONS FOR INSTRUCTION}

It is clear from prior research that thinking about and utilizing integration while working through physics problems is a challenge for students [1-7]. This paper has shown that common texts used for physics instruction tend to emphasize the adding up pieces and procedural representations over the perimeter and area and antiderivative presentations. It is clear that there is a disconnect between the learning goals of mathematics and physics courses where integration is concerned, and it is likely that most physics instructors are unaware of this difference.

The research suggests that physics instructors cannot take for granted that students will understand the integral as an expression of a sum. The interpretation of an integral as a sum needs to be made explicit to students the way Knight does throughout the text as we cannot expect them to have been exposed to this in their mathematics courses. In introductory electricity and magnetism for example, there are many instances in which there is no area to speak of when using integration to solve a problem. Being able to articulate the integral as a sum in these problems is not only helpful but a necessary step to success.

\section{AKNOWLEDGEMENTS}

This work is supported by the NSF through grant PHYS1405616; opinions, findings, and conclusions are those of the author and do not necessarily reflect the views of the NSF. The lead author would like to thank the Black family fellowship for additional support. 
[1] Bajracharya, R. R. (2015). Student application of the fundamental theorem of calculus with graphical representations in mathematics and physics. Dissertation Abstracts International: Section B: The Sciences and Engineering, 76(4-B(E)). Retrieved from http://search.ebscohost.com/login.aspx?direct $=$ true $\& \mathrm{db}=\mathrm{ps}$ yh\&AN=2015-99200-250\&site $=$ ehost-live

[2] Doughty, L., McLoughlin, E., \& van Kampen, P. (2014). What integration cues, and what cues integration in intermediate electromagnetism. American Journal of Physics, 82(11), 1093-1103. https://doi.org/10.1119/1.4892613

[3] Hu, D., \& Rebello, N. S. (2013). Understanding student use of differentials in physics integration problems. Physical Review Special Topics - Physics Education Research, 9(2), 1-14. https://doi.org/10.1103/PhysRevSTPER.9.020108

[4] Pollock, E. B., Thompson, J. R., \& Mountcastle, D. B. (2007). Student understanding of the physics and mathematics of process variables in $\mathrm{P}-\mathrm{V}$ diagrams. AIP Conference Proceedings, 951, 168-171. https://doi.org/10.1063/1.2820924

[5] Von Korff, J., \& Rebello, N. S. (2012). Teaching integration with layers and representations: A case study. Physical Review Special Topics - Physics Education Research, 8(1), 1-16. https://doi.org/10.1103/PhysRevSTPER.8.010125

[6] Sealey, V. (2006). Definite integrals, riemann sums, and area under a curve: What is necessary and sufficient. In $P M E$ NA Proceedings, 2(1991), 46-53.

[7] Jones, S. R. (2013). The Journal of Mathematical Behavior Understanding the integral : Students' symbolic forms. Journal of Mathematical Behavior, 32(2), 122-141. https://doi.org/10.1016/j.jmathb.2012.12.004

[8] Jones, S. R. (2015a). Areas, anti-derivatives, and adding up pieces: Definite integrals in pure mathematics and applied science contexts. Journal of Mathematical Behavior, 38, 928. https://doi.org/10.1016/j.jmathb.2015.01.001

[9] Jones, S. R. (2015b). The prevalence of area-under-a-curve and anti-derivative conceptions over Riemann sum-based conceptions in students' explanations of definite integrals. International Journal of Mathematical Education in Science and Technology, 46(5), 721-736.

[10] Sealey, V. (2014). The Journal of Mathematical Behavior A framework for characterizing student understanding of Riemann sums and definite integrals. Journal of Mathematical Behavior, 33, 230-245. https://doi.org/10.1016/j.jmathb.2013.12.002
[11] Sherin, B. L. (2010). How Students Understand Physics Equations How Students Understand Physics Equations. 0008(January 2013), 37-41. https://doi.org/10.1207/S1532690XCI1904

[12] Meredith, D. C., \& Marrongelle, K. A. (2008). How students use mathematical resources in an electrostatics context. American Journal of Physics, 76(6), 570-578. https://doi.org/10.1119/1.2839558

[13] Cui, L., Sanjay, R., \& Fletcher, P. (2006). Transfer of Learning from college calculus to physics. Proceedings of the National ..., (2004), 1-10. https://doi.org/10.1080/0020739X.2014.1001454

[14] Tulip, D., \& Cook, A. (1993). Teacher and student usage of science textbooks. Research in Science Education, 23(1), 302-307. doi:10.1007/bf02357074(Bajracharya, 2015; Doughty, McLoughlin, \& van Kampen, 2014; Hu \& Rebello, 2013; Pollock, Thompson, \& Mountcastle, 2007; Sealey, 2014; Von Korff \& Rebello, 2012)

[15] Halliday, D., Resnick, R., \& Walker, J. (2018). Fundamentals of physics. Milton, Qld: John Wiley \& Sons Australia.

[16] Giancoli, D. C. (1988). Physics for scientists and engineers with modern physics. Englewood Cliffs, NJ: Prentice-Hall.

[17] Young, H. D., \& Freedman, R. A. (2003). University physics with modern physics. Menlo Park, CA: Benjamin Cummings.

[18] Knight, R. D. (2017). Physics for scientists and engineers: A strategic approach. Boston: Pearson.

[19] Serway, R. A., \& Jewett, J. W. (2016). Physics for scientists and engineers with modern physics / Raymond A. Serway, John W. Jewett, Jr. Australia: Cengage Learning.

[20] Bajracharya, R. R., \& Thompson, J. R. (2016). Analytical derivation: An epistemic game for solving mathematically based physics problems. Physical Review Physics Education Research, 12(1), 1-21. https://doi.org/10.1103/PhysRevPhysEducRes.12.010124

[21] Stewart, J. (2013). Essential calculus: Early trancendentals. Australia: Brooks/Cole, Cengage Learning. 